\title{
Electronic Differential Control of Independent Wheel Drive NEV using Drive Current Balancing Strategy
}

\author{
Pranjal Barman, Santanu Sharma
}

\begin{abstract}
This paper presents the design and real-time implementation of an electronic differential speed control system (EDSC) for a neighborhood electric vehicle (NEV) with a decentralized power train configuration. The EDSC supervises the desired speed variation of the drive motors of the vehicle at various turning trajectories. The core focus of this design is to reduce the system complexity, computation delay and design expenses with an aid of a newly proposed drive current balancing algorithm (DCBA). The embedded DCBA based EDSC allocates the necessary torque to each wheel solely depending on the motor current variables. Thus, it eliminates the necessities of typical feedback variables-steering and speed to control the EDSC. The developed system performance is being investigated in situ real time on-board experiment. Results in the context of response time, design simplicity and performance reveal the effectiveness of the proposed framework.
\end{abstract}

Keywords: Electronic Differential (ED); Current Balancing; Neighborhood Electric Vehicle (NEV); Permanent Magnet DC Motor (PMDC).

\section{INTRODUCTION}

Electric Vehicles (EV) have been garnering wide attention over traditional combustion engine based vehicles due to the growing concerns of continuously depleting fossil fuel and environmental safety [1]-[5]. Despite having several advantages, the EVs are still competing against the whole varieties of Internal Combustion Engine (ICE) vehicle alternatives with their respective strengths and limitations. The EV performs well as a quick and efficient mass transit system or as a small sized lightweight personal vehicle that demands the requirement of a new class of vehicle, namely, Neighborhood Electric Vehicle (NEV) [6] .

The NEV is required to be simple, affordable and efficient that could provide the necessary transportation within a close community wherein speed and range restrictions are not major concerns [7]. It could further reduce the traffic congestion and public parking spot. In typical EV, a single drive motor is mounted between the driving wheels through reduction gear and mechanical differential system [8], [9]. Such design considerably increases the overall mass of the vehicle along with the in-house peripherals [10] for which reduction of the vehicle mass is an important criteria.

Revised Manuscript Received on February 05, 2020.

* Correspondence Author

Pranjal Barnman, Department of Electronics and Communication Engineering. Technology from Tezpur University, Assam, India.

Santanu Sharma, Associate Professor. electronics and communication engineering from Tezpur University, Tezpur, India,

(C) The Authors. Published by Blue Eyes Intelligence Engineering and Sciences Publication (BEIESP). This is an open access article under the CC BY-NC-ND license (http://creativecommons.org/licenses/by-nc-nd/4.0/)
Thus, independently equipped motor-wheels of the vehicle are an effective solution. Such configuration requires embedding of individual smaller motors to be mounted with each wheel. It provides potential advantages in terms of flexibility, controllability and responsiveness over typical design [11], [12]. The major bottleneck in such design is the precise torque distribution among the wheels so as to enhance the performances [13].

In order to outweigh the challenge of the typical design electronic differential (ED) control plays a vital role. It distributes necessary torque to the wheels in such a manner that the rotation of outer and inner wheels is synchronized over different curvilinear paths. Unlike the mechanical differential, it provides significant improvements in terms of responsiveness and efficiency. Nevertheless, it relies on various feedback signals from motor that to be regulated strictly in order to avoid faulty operations. A careful approach with well-defined framework could outreach the shortcomings of the EVs with ED and make it potential in industrial prospective. Despite significant contributions in previously published works there is a lacking in cost effective solutions of ED without trading off the design simplicity, stability and robustness [14], [15]. The method [16] employed linear synchronization to derive the wheel reference speed using Ackermann steering geometry. It used velocity and steering angle feedbacks to control the speed difference of inner and outer wheel during a turning maneuver. Work in [17] adopted a direct-torque control scheme with same objective. Method in [18] employed speed ratio of the driving wheels as a control variable and improved the transient response of the system. Mutoh et al. in [19] adopted a method by estimating adequate lateral forces for wheel revolution based on multiple feedback variables in order to enhance the off-road driving performance. All the aforementioned strategies are based on predefined static steering geometry as well as several online feedback parameters. Hence, the system complexity and computational burden increases due to usage of large feedback parameters result in requirement of high speed controller that increase the cost burden. Moreover the adoption of static steering geometry limits the reliability of the system as it does not account the effect of centripetal force on wheels. The existing literature are lacking in addressing the appropriate ED strategies for NEV that meet the requirement like simple, tunable, robust and efficient system at limited speed range. 
This work embeds a new EDSC by adopting a drive current balancing algorithm (DCBA) using offline motor torque speed characteristics. The proposed method eliminates the requirement of steering wheel feedback and that of predefined geometry. It does not require expensive sensors and high computation power thereby reduces the design expenses, system complexity and robustness. The main contribution of our work is summarized as follow:

1. Design and analysis of the DCBA for an electronic differential specifically developed for the NEV;

2. The algorithm is implemented in offline mode using simulated data to investigate performance and utility for real-time applications. The validated algorithm is then embedded to high performance interface board with inbuilt microcontroller using which the developed NEV has been tested successfully;

3. The results obtained from the simulation as well as the on-board validation are outlined and comprehensively discussed with comparison to the traditional steering feedback based system.

\section{THEORY AND METHODOLOGY}

The aims of employing ED in NEV is to provide adequate torque and speed to the inner and outer wheel at various cornering maneuver. Fig.1 shows the EV configuration with static steering geometry. Herein, $v$ is velocity of the Centre of gravity; the inner and outer wheel velocities must be directly proportional to the distance of the wheels to the turning center as follows:

$$
\begin{aligned}
& \frac{v_{\text {in }}}{R_{\text {in }}}=\frac{v}{R}=\frac{v_{\text {out }}}{R_{\text {out }}} \\
& R_{\text {in }}+R_{\text {out }}=2 R
\end{aligned}
$$

Wherein, $v_{\text {in }}$ and $v_{\text {out }}$ are inner and outer motor velocity, and $R, R_{\text {in }}$ and $R_{\text {out }}$ are turning radius of the mass center, inner wheel turning radius and outer wheel turning radius respectively. As defined in [20], the performance of driving motor in terms of motor parameters during cornering can be expressed as follows:

$$
\begin{aligned}
& V_{\text {in }}=K_{e} \omega_{\text {in }}+L_{a} \frac{d i_{\text {in }}}{d t}+R_{a} i_{\text {in }} \\
& V_{\text {out }}=K_{e} \omega_{\text {out }}+L_{a} \frac{d i_{\text {out }}}{d t}+R_{a} i_{\text {out }}
\end{aligned}
$$

Here $V_{\text {in }}$, and $V_{\text {out }}$ are inner and outer motor voltage, and $i_{\text {in }}$ and $i_{\text {out }}$ are inner motor and outer motor currents, $\omega_{\text {in(out) }}$ is the inner (outer) angular velocity, $K_{e}$ is the back emf constant. Solving Eq.(3) and Eq.(4) for equal voltage level, the current $i_{d}$ is evaluated as follows:

$$
K_{e}\left(\omega_{\text {in }}-\omega_{\text {out }}\right)+L_{a} \frac{d\left(i_{\text {in }}-i_{\text {out }}\right)}{d t}+R_{a}\left(i_{\text {in }}-i_{\text {out }}\right)=0
$$

Defining $i_{d}=\left(i_{\text {in }}-i_{\text {out }}\right)$ and $\Delta \omega=\left(\omega_{\text {in }}-\omega_{\text {out }}\right)$, the above Eq.(5) is further simplified as

$$
\begin{aligned}
& K \Delta \omega+L_{a} \frac{d i_{d}}{d t}+R_{a} i_{d}=0 \\
& i_{d}=\frac{K_{e} \Delta \omega}{R_{a}}\left(e^{-\frac{R_{a}}{L_{a}} t}-1\right)
\end{aligned}
$$

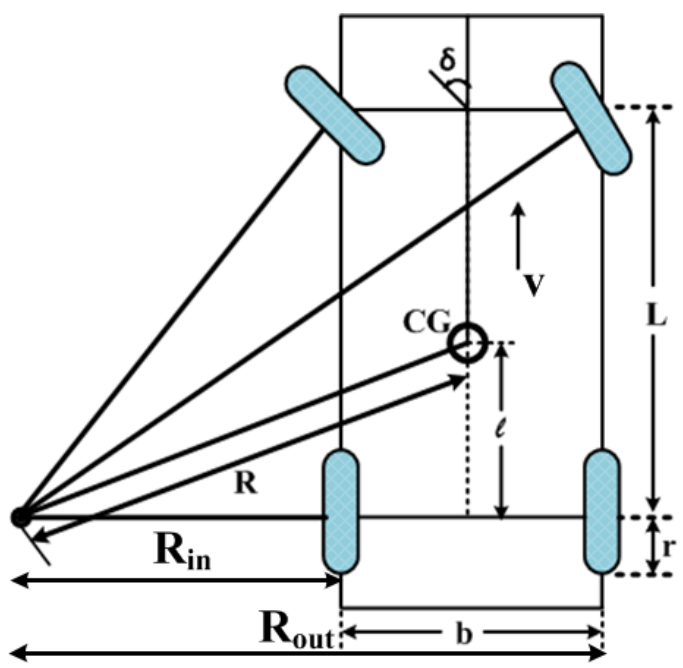

Fig. 1.EV configuration with Ackermann steering geometry where $R$ is the radius of turning with respect to the centre of gravity (CG). Here $L, b, l, r$ and $v$ indicate respectively
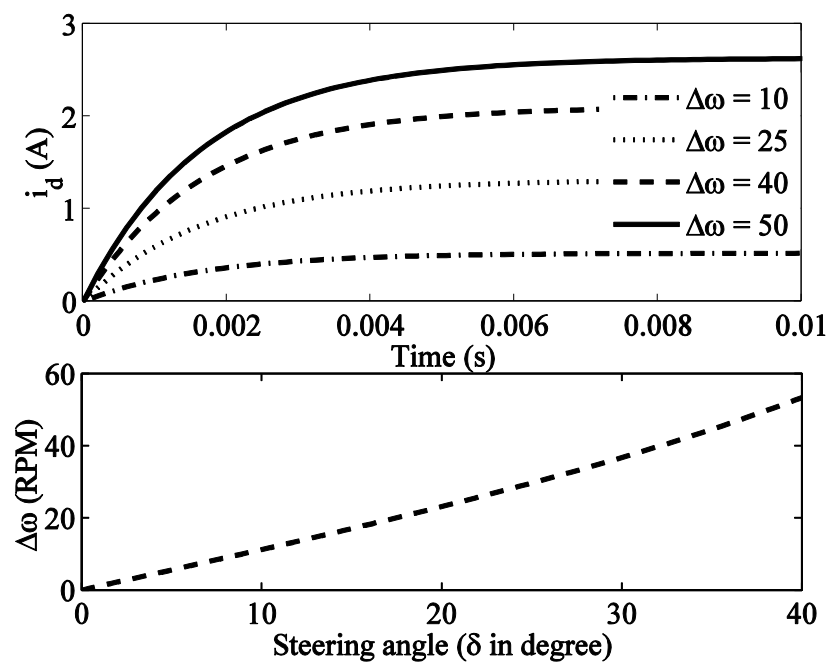

Fig. 2.Transient response of current difference at various wheel speed differences and the respective steering angles

As is evident from Eq.(7) that the current difference between inner and outer motor is a proportional to the speed difference of the respective wheels in addition to the exponential factor. The transient response of the current difference for different $\Delta \omega$ at 10, 25, 40 and 50 illustrated in Fig.2 which reveals the consequences of Eq.(7). The respective steering angles for theoretical wheel speed differences are mentioned in $\delta$ versus $\Delta \omega$ plot. Nevertheless, the practical values of current difference will be slightly higher than that of theoretical values presented in Fig. 2 which is due to the effects of the centrifugal force. Thus, it is seen that a new differential mechanism can be proposed taking the idea of linearly dependent parameters as outlined in Eq.(7) in order to avoid using steering and speed feedback. It could ensure simplicity of the design by avoiding theoretical bottleneck of the system. 
Assuming equal current in Eq.(3)-(4) and substituting in Eq.(1) -(2) [21], the voltage difference is evaluated as below.

$$
V_{\text {in }}-V_{\text {out }}=K_{e}\left(\omega_{\text {in }}-\omega_{\text {out }}\right)=K_{e} \omega \frac{\left(R_{\text {in }}-R_{\text {out }}\right)}{R}
$$

Eq.(8) illustrates that the required differential action for a turning maneuver for balanced the current levels in both inner and outer drive motors. In order to balance the current, the armature voltages need to be changed up to desired levels that need to be employed the DCBA algorithm which compensates the current error by using motor characteristics. The proposed method delivers equal amount of torque to the driving wheels irrespective of the direction of turning. An iterative change of voltage variables according to the DCBA control algorithm successively settles the current error during the curvilinear motion of the NEV. This results in the differential wheel rotation without the active involvement of steering angle feedback.

\section{CONTROLLER DESIGN}

The DCBA is realized to compensate the voltage variable based on the dynamic error function of the motor current during vehicle trajectory. The prerequisite parameter of the DCBA algorithm is the maximum current error $\left(\mathrm{e}_{\max }\right)$ and gain $(K)$. In order to determine these parameters, a series of real-time experiments are being conducted without implementing the differential system into the NEV. Subsequently, the $\mathrm{e}_{\max }$ is calculated by emulation of current difference at multiple turning angles over a spiral course of turning as depicted in Fig.3. The current error with respect to the steering angle at different wheel speed is shown in Fig.4. It is observed that the $e_{\max }$ is around $\pm 10 \mathrm{~A}$, which occurs at 115 RPM. The current difference is seen reduced at higher vehicular speed. This is due to the fact that the influence of the centrifugal force, the inner wheel experiences less traction force than the outer wheel. Consequently the drive motor current of the inner wheel decreases at higher vehicle speed. The controller has been designed in such a way that, if the current error lies below the maximum value $e_{\max }$, the control logic works in linear region. The linearity constant $K$ is determined from the theoretical analysis and experimental validation of the NEV. That is to determine $K$, a right turning maneuver is selected over the same spiral course mentioned in Fig.3. Then a theoretical analysis is examined to find out the inner and outer wheel speed with respect to the steering angle following [15]. Then, a set of experiments is conducted by using various values of $K$ which is presented in Fig.5. As is evident, at $K=8$, the theoretical curve is closely matched with the experimental data and therefore, it is considered as the linearity constant during implementation of DCBA algorithm in the NEV.

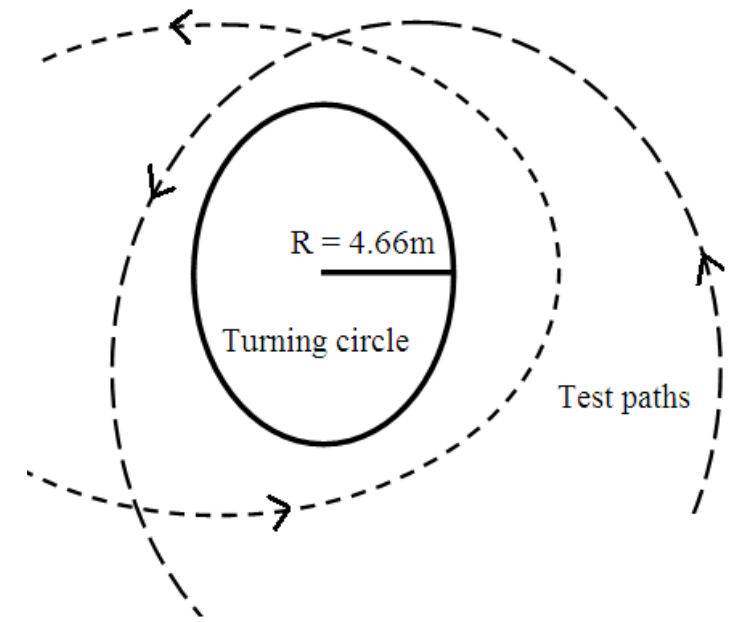

Fig. 3.Test path for $\boldsymbol{e}_{\max }$ measurement

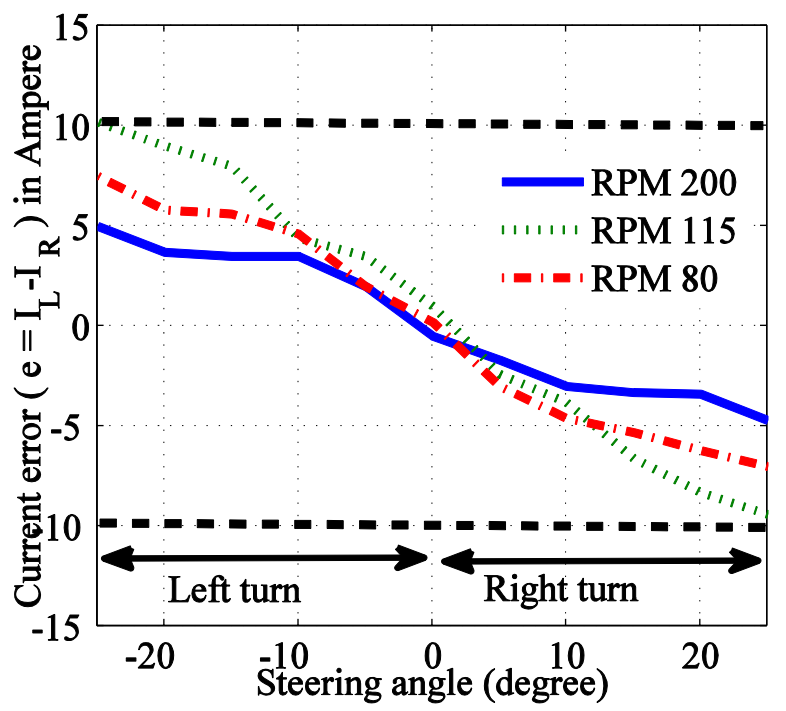

Fig. 4.Current error at various steering angle

Fig.6 shows the DBCA algorithm used herein. The output of the DCBA is the correction factor $\mathrm{Z}$ which is to be added or subtracted to the control voltage for each drive motors. This process work in iterative manner for every control cycle till the error function converges to zero.

The algorithm is verified by software simulation with the following assumptions. i). Left and right motor currents are assumed to be changed at different steering angle $(\delta<0$ : left turning, $\delta>0$ : right turning), ii). Drive motor characteristics are identical and iii). Acceleration command is fixed for a particular simulation.

The simulation results are presented in Fig.7. It is seen that during a turning maneuver (left turning), the left motor current starts increasing compared to that of right motor. As a consequence, the factor $\mathrm{Z}$ of the DCBA changes the input voltage of the motors as per the requirement. It is seen that during the turning maneuver, the control voltage of the right motor increases compared to the driver assisted input voltage. On the other hand, the control voltage of the left motor decreases compared to the input voltage. 


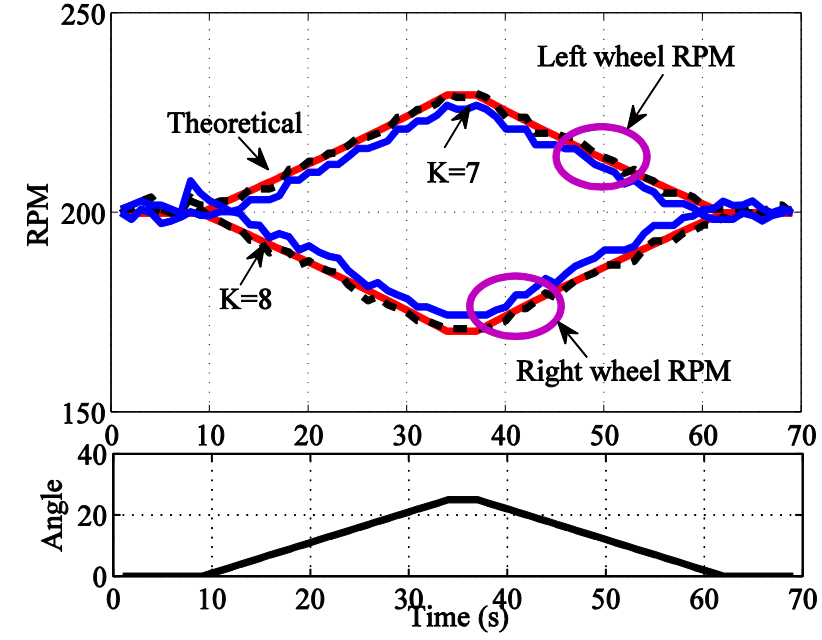

Fig. 5.Estimation of $K$ value using experimental data

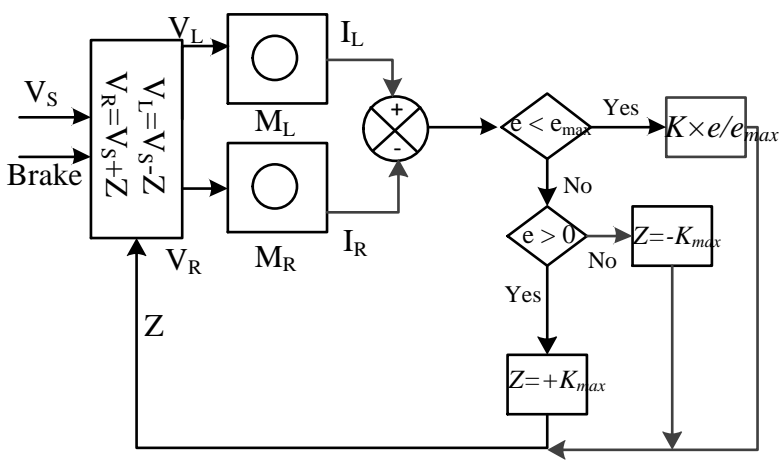

Fig. 6.DCBA based ED system

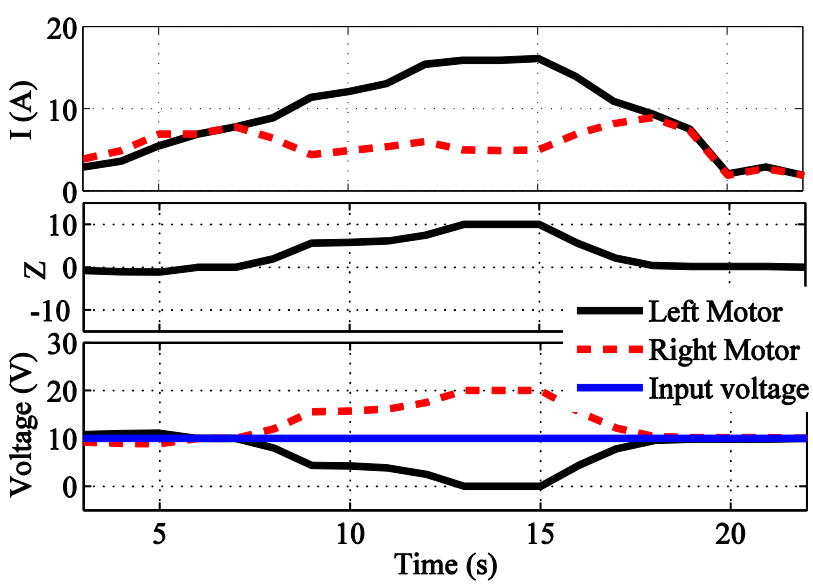

Fig. 6.Simulation result of DCBM algorithm

Thus, the proposed algorithm works effectively in electronic differential action mode that fulfills the needs. However, the control voltages of the motors are limited by the motor characteristics as illustrated in Fig.8 which was analyzed from the method presented in [22].

\section{SYSTEM IMPLEMENTATION AND ANALAYSIS}

The performance of the proposed differential is examined using a newly developed NEV prototype. The NEV uses a rear independent wheel-drive system relying on the permanent magnet DC motor (PMDC) driving principle, wherein each motor is connected to one of the wheels through pulley sheave-belt coupling. The coupling reduces the motor

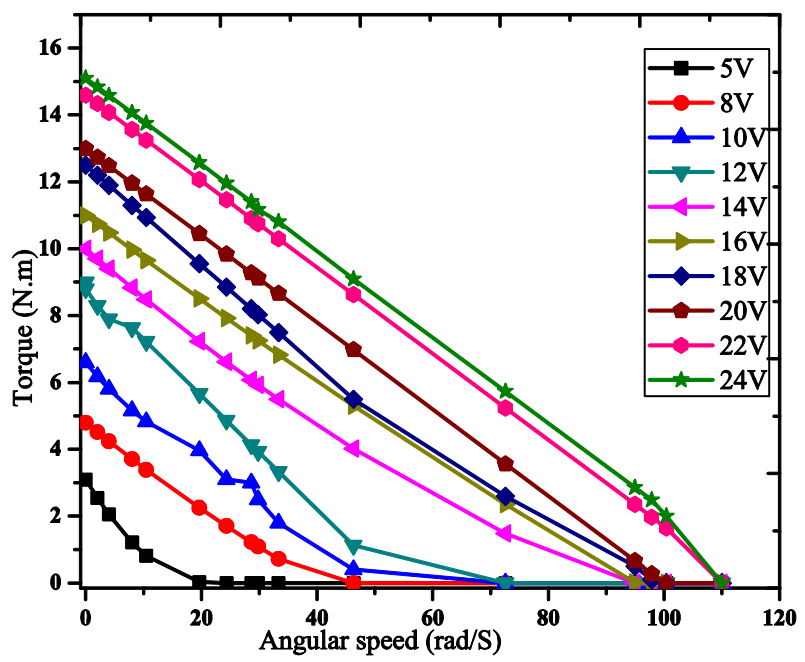

Fig. 7.Motor Torque-Speed Characteristics

Table- I: Specification of PMDC drive components

\begin{tabular}{|l|l|l|}
\hline \multicolumn{1}{|c|}{ Components } & \multicolumn{1}{|c|}{ Type } & \multicolumn{1}{c|}{ Specification } \\
\hline Motor & PMDC & $\begin{array}{l}1 \mathrm{HP}, 24 \mathrm{~V}, 3000 \mathrm{RPM}, \mathrm{\eta}=78 \%, \\
\mathrm{~L}_{\mathrm{a}}=200 \mu \mathrm{H}, \mathrm{R}_{\mathrm{a}}=0.15 \Omega, \\
\mathrm{K}_{\mathrm{e}}=13.25 \mathrm{~V} / \mathrm{RPM}\end{array}$ \\
\hline Battery & Lead Acid & $12 \mathrm{~V}, 26 \mathrm{Ah}$ \\
\hline DC chopper & --------- & $24 \mathrm{~V}, \mathrm{I}_{\max }=180 \mathrm{~A}, \mathrm{f}=8 \mathrm{kHz}$ \\
\hline Current sensor & WCS1600 & $100 \mathrm{~A}, \mathrm{~S}=22 \mathrm{mV} / \mathrm{A}$ \\
\hline
\end{tabular}

to wheel speed by 1:10 gear ratio.

A high power PMDC chopper is designed to actuate the motors over a wide torque-speed range as shown in Fig.9. PMDC-driver components are outlined in Table I. A parallel MOS switching is configured to reduce excessive on-state heat dissipation of the system. The module with in-built microcontroller controls the motors based on the sensor feedback, DCBA algorithm. The offsets associated with the sensors and respective drive circuits that effect system responses, have been nullified prior to validation. The DC chopper mounted in the front panel with necessary sub-circuits as shown in Fig.10. The back panel of the vehicle consists of battery and freewheeling section shown in Fig.11. The complete prototype of the NEV is shown in Fig.12. An online data acquisition system with display interfacing is developed and embedded into the NEV. During driving, the acquisition and display unit collects various online transient signals in order to evaluate the ED performance.

The current is the major control variable in the DCBA strategy. Therefore, the motor currents (i) of the NEV are measured in every maneuvering point of the trajectory path. This is done to ensure the predefined constraints of the system as well as the improvement of the DCBA scheme. It is essential to note that theoretically there should be identical value of $i$ for each driving motor on straight path over a flat surface. However, in real scenario, a small difference is observed which is presumably due to inherent dissimilarities of the motor components, circuit dissimilarities and difference mechanical coupling.

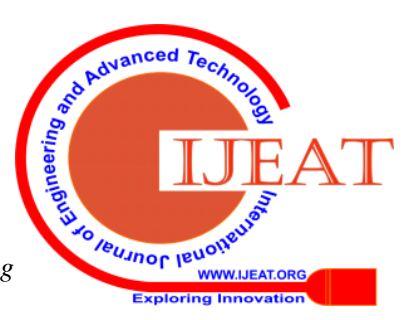


This problem has been resolved by setting a current threshold and subsequently, other important parameters such as wheel speed, input voltage and steering angle are being measured throughout the drive trip. A drive trip road map is selected for the experiment which is shown in the Google Map trajectory as illustrated in Fig.13. Experiment has been carried out several times with same settings to ensure the repeatability of the system and relevant results are demonstrated.

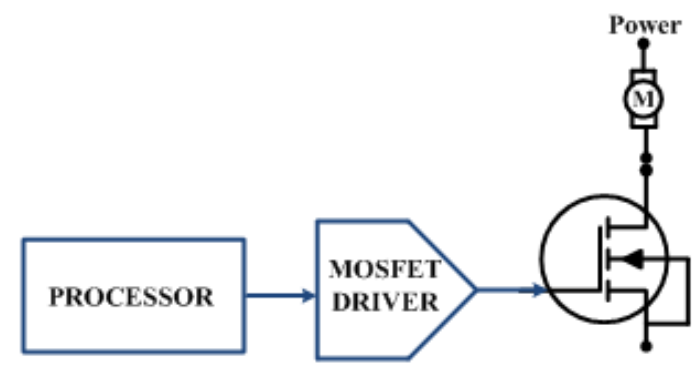

Fig. 8.Schematic of NEV drive control module

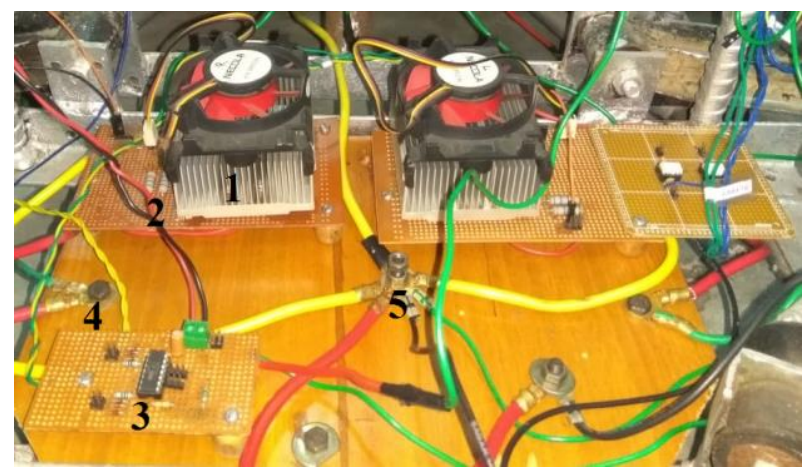

Fig. 9.PMDC driver circuit (1-MOSFET array with heat sink and cooler, 2-Snubber, 3-Gate driver, 4-Port for motor connections, 5-Power ground)

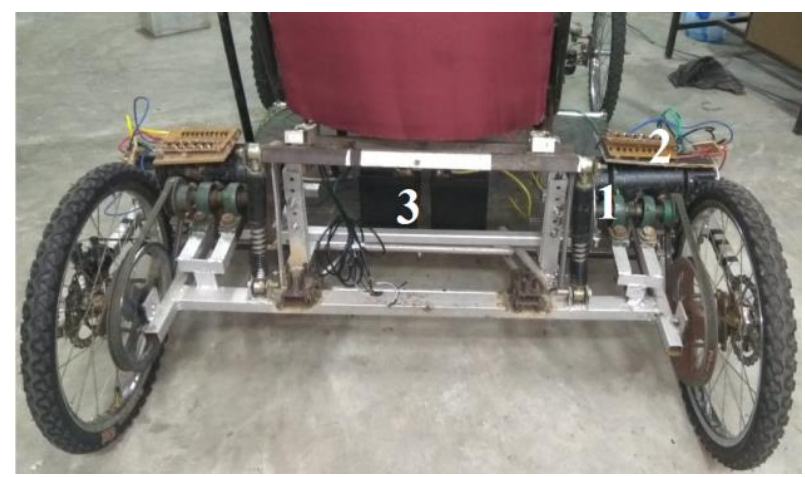

Fig. 10. Back-panel of NEV (1-Motor, 2-Freewheeling, 3-Battery)

For two control wheels, two difference responses of current, voltage and speed are measured in the trip. The vehicle accelerates from resting position A to position B and gradually makes a left-turn towards C. From A to B; steering angle $\delta=0$ while it is maximum negative at $\mathrm{B}$. It is worth mentioning that, in this experiment the negative indicates the left turning and positive indicates the right turning of the NEV. The process will remains same until the vehicle approaches the right turns at point D. However, at D, the process will reverse in order to change the course of the direction of NEV and finally stops at the termination point E.

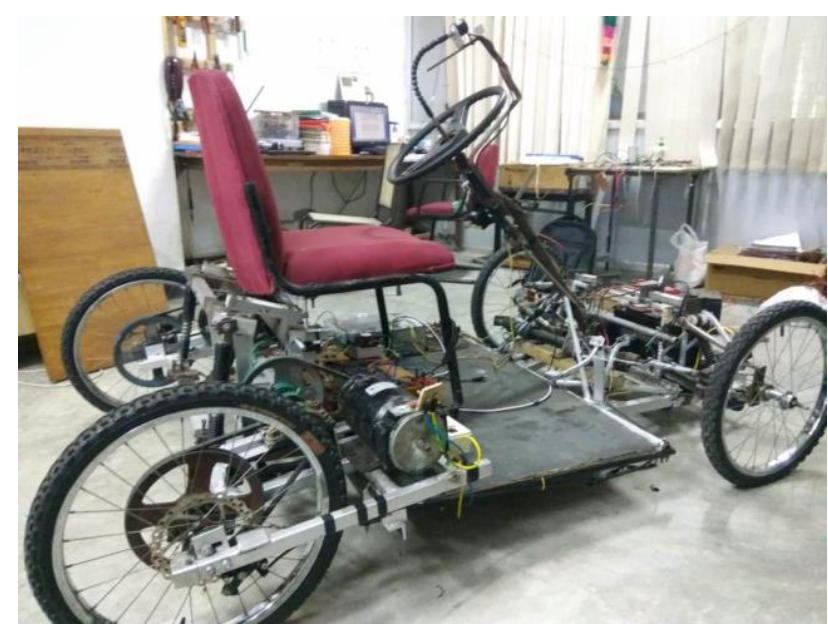

Fig. 11. Developed NEV prototype for various experiments

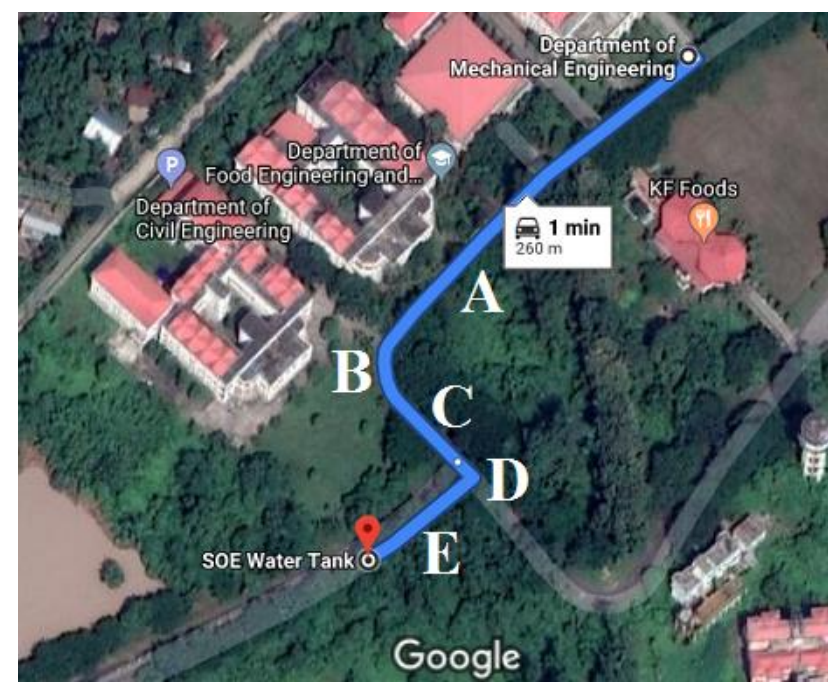

Fig. 12. Visual description of trajectory path (A-E) in Google map image

It is worth noting that the proposed DCBA aims to balance the current in order to activate the differential action throughout the trip irrespective of direction of turn. The torque responses therefore at various cornering trips are essentially proportional to that of the current. However, there is an unavoidable current fluctuation occurs due to the sudden transfer of tractive effort between the driving wheels in a maneuver. The relation of current and torque of PMDC is linear, thus the DCBA tries to reduce the current error function by symmetrically changing their respective armature voltages. In case of left turning, left wheel is the inner wheel and right wheel is the outer wheel. This will be reversed during the right course of cornering. The NEV, therefore, shows a counter changing of RPM between the left and right wheel during the cornering maneuvers.

\section{V.RESULTS AND DISCUSSIONS}

The integrity of proposed method lies in eliminating the conventional steering and speed feedback from the control strategy and in improving the system response. Therefore, in order to evaluate complete

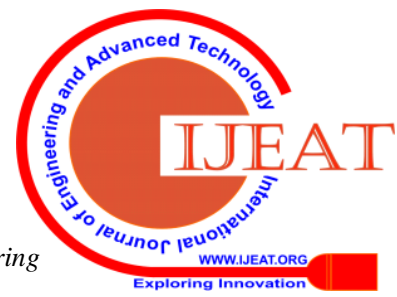




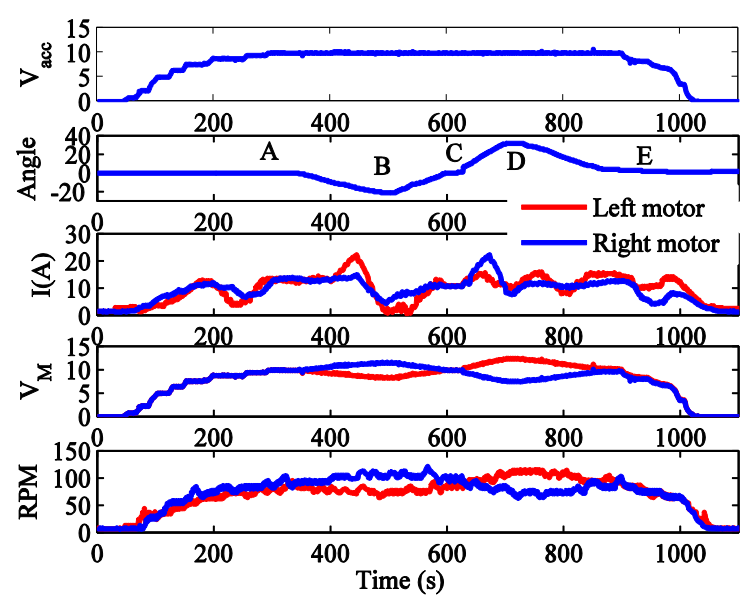

Fig. 13. Control parameter variations during the drive trip with ED over the specified Google map trajectory shown in Fig.13

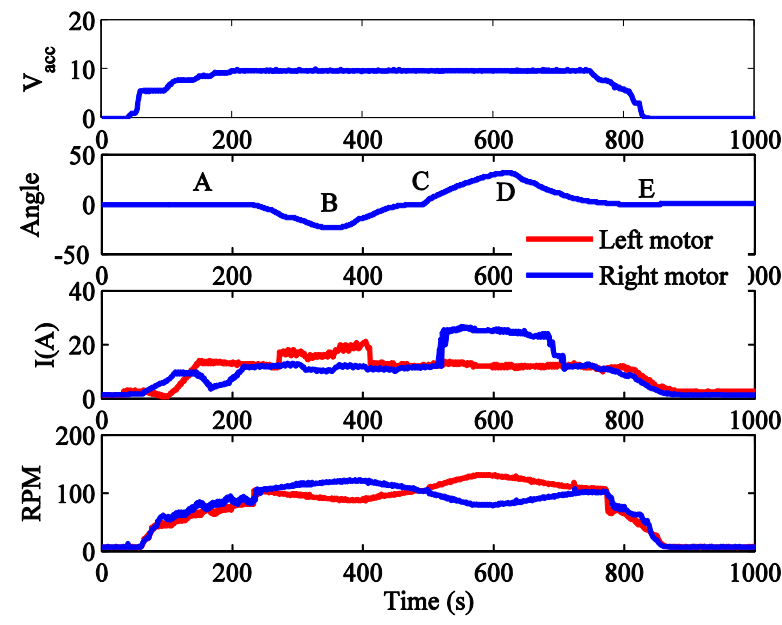

Fig. 14. Control parameter variations during the drive trip without the ED

performance analysis, the validation has been carried out over the aforementioned trajectory as well as a spiral course with necessary maneuvering. Fig.14 shows the various parameters observed from the real road testing of the NEV. It is seen that the drive current is balanced due to the changes of control voltage $\left(V_{M}\right)$ at various turning of the drive trip. The symmetric variation of motor control voltage as well as wheel RPM demonstrates the successful working of the proposed ED. In order to find out the effectiveness of the vehicle, another experiment has been conducted without implementing the ED into the NEV. It is shown in Fig.15. Then a spiral course is selected with the turning radius as mentioned in Fig.3. Another set of experiments have been conducted with and without the ED over the spiral drive trajectory. The motor parameter variations with and without ED during the spiral course is presented in Fig.16 and Fig.17 respectively. It is noted that during the spiral drive trip, only left cornering performances are presented in this article. The right cornering performance is just opposite to that of the left cornering performance. However, for the ease of explanation, the possible nominal dissimilarities between the left and right cornering performances are ignored. A comparison of total motor power has been evaluated from the aforementioned results which are shown in Fig.18.

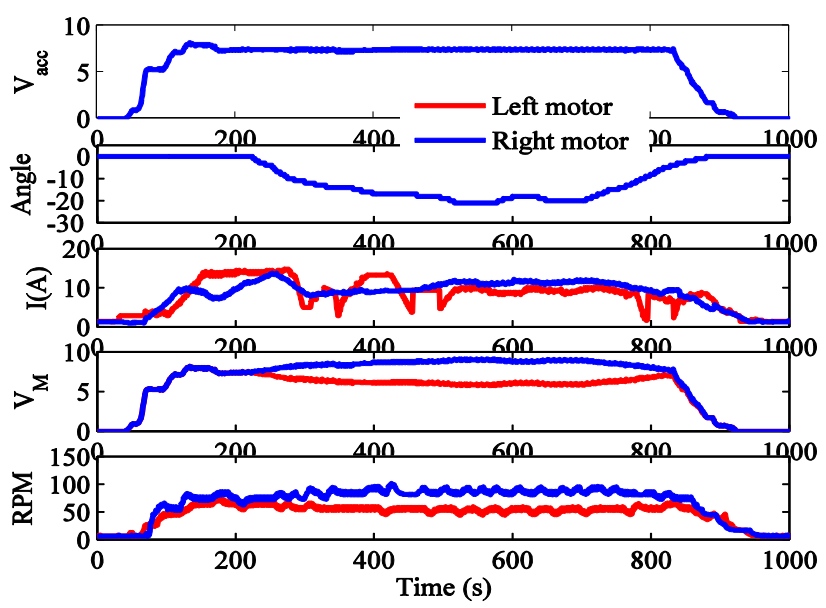

Fig. 15. Control parameter variations during the spiral drive trip with ED

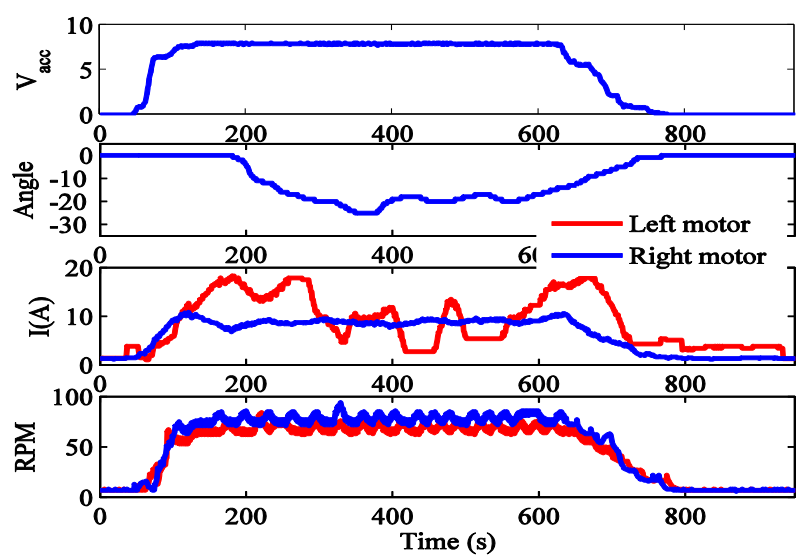

Fig. 16. Control parameter variations during the spiral drive trip without ED

The summation of the average drive motor power is tabulated in Table.II. It is seen that the total motor power consumption without ED is higher than that of with the ED. A comparative analysis is carried out to show the merit of the proposed ED over the typical steering feedback based approach. A steering feedback based algorithm has been implemented into the $\mathrm{NEV}$ and perform the previously explained road tests and accordingly, the power requirement for both the cases is calculated. The motor power consumption of the NEV in both the cases is illustrated in Fig.19 which indicates that the power consumption conventional method is more than that of the proposed method. In addition, some methods akin to our analysis is also highlighted in Table.III in the context of some relevant parameters which evinces the effectiveness of the proposed method.

\section{CONCLUSION}

The study focused on development of ED based NEV and performance evaluation with a proactive DCBA control algorithm. The proposed system do not actively use the steering feedback and velocity sensors thus simplifies the system complexity and reduces the cost. Furthermore, the current feedback concept makes the system faster, accurate and effectively applicable in NEV driving. Several on-road experiments were conducted to validate the system. 
The promising performance as seen from the comparative analysis reveals the improvements in reduction of overall power consumption of the NEV. Even though the aforesaid study is merely based on PMDC motor technology, the strategy could essentially be extended for AC or BLDC motors. The advanced AC motor control method namely Field Orientation Control (FOC) requires phase current observer to estimate the rotor position. On the basis of estimated rotor position, the FOC controller generates necessary input voltage to the motor. Likewise, the DCBA strategy could be adopted in FOC mechanism so as to regulate the motors according to various maneuvering demand. There could be several opportunities to further improve the proposed method. Nevertheless, by considering the limitations of the NEV driving environment, the rationality of the proposed article excludes the analysis over off-road and undulating road surfaces.
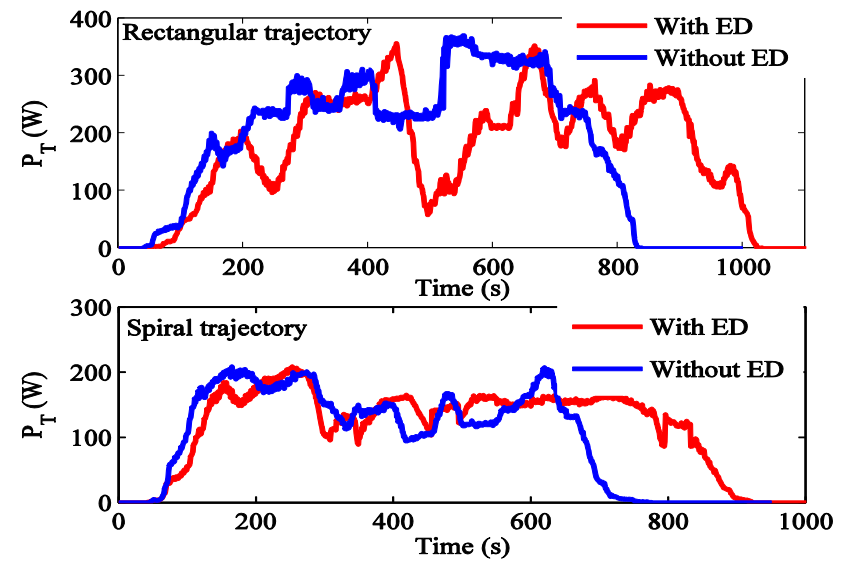

Fig. 17. Sum of the average power of the motors with and without ED over the rectangular and spiral drive trajectory

Table- II: Average motor power

\begin{tabular}{|l|l|l|}
\hline Drive trajectory & \multicolumn{1}{|c|}{ With ED } & Without ED \\
\hline Rectangular & $276 \mathrm{~W}$ & $336 \mathrm{~W}$ \\
\hline Spiral & $191 \mathrm{~W}$ & $203 \mathrm{~W}$ \\
\hline & $\begin{array}{l}\text { Conventional } \\
\text { Method }\end{array}$ & Proposed Method \\
\hline Rectangular & $197 \mathrm{~W}$ & $160 \mathrm{~W}$ \\
\hline
\end{tabular}

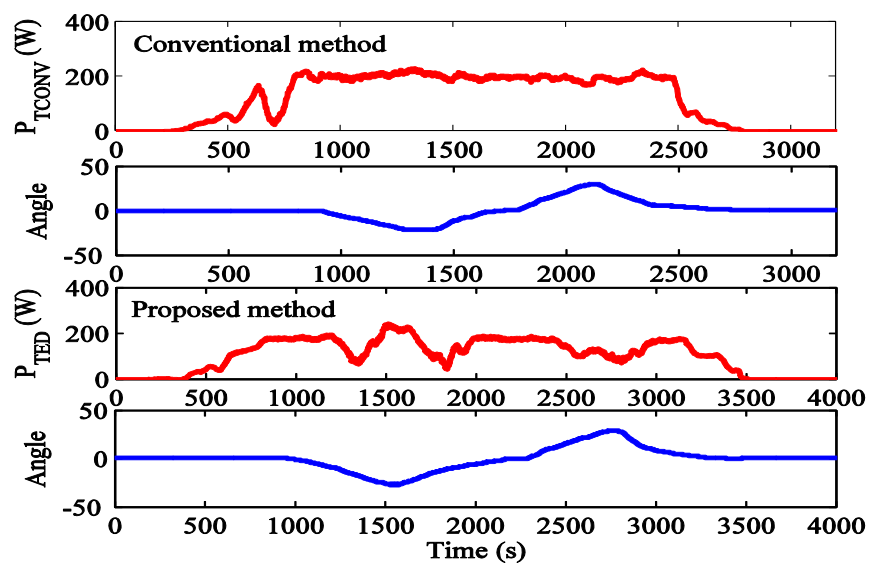

Fig. 18. Motor power consumption comparison with the conventional metod
Table- III: Relevant comparisons of DCBM with conventional method

\begin{tabular}{|l|l|l|}
\hline Comparison & DCBM method & $\begin{array}{l}\text { Conventional } \\
\text { method }\end{array}$ \\
\hline Response time & $9.5 \mathrm{~ms}$ & $17 \mathrm{~ms}$ \\
\hline Power consumption & Lower & Higher \\
\hline Sensor used & Current sensor & $\begin{array}{l}\text { Angle, speed and } \\
\text { current sensors }\end{array}$ \\
\hline $\begin{array}{l}\text { Computational } \\
\text { complexity }\end{array}$ & $\begin{array}{l}\text { Moderate with less } \\
\text { feedback parameters }\end{array}$ & $\begin{array}{l}\text { Higer with } \\
\text { multi-sensor interfaces }\end{array}$ \\
\hline
\end{tabular}

\section{ACKNOWLEDGMENT}

The authors would like to thank the Department of Electronics and Communication Engineering, Tezpur University, India for providing design facilities. The authors would also like to acknowledge Chaitanya Borah, Surabhi Agrawal and Sujan Neroula for their cooperation during the development of the prototype.

\section{REFERENCES}

1. T. Ma, and A. M. Osama, 'Optimal charging of plug-in electric vehicles for a car-park infrastructure', IEEE Trans. Ind. Appl., Vol. 50, No. 4, pp.2323-2330, 2014.

2. A. Y. Saber, and G. K. Venayagamoorthy, 'Plug-in vehicles and renewable energy sources for cost and emission reductions', IEEE Trans. Ind. Electron., Vol. 58, No. 4, pp.1229-1238, 2011.

3. M. Yildirim, M. C. Catalbas, A. Gulten, and H. Kurum, 'Computation of the Speed of Four In-Wheel Motors of an Electric Vehicle Using a Radial Basis Neural Network',Engineering, Technology Applied Science Research., Vol. 6, No. 6, pp.1288-1293, 2016.

4. C.H. T Lee,K. T.Chau, andC. Liu, 'Design and analysis of an electronic-geared magnet less machine for electric vehicles', IEEE Trans. Ind. Electron., Vol. 63, No. 11, pp.6705-6714, 2016.

5. A. Emadi, Y. J. Lee, and K. Rajashekara, 'Power electronics and motor drives in electric, hybrid electric, and plug-in hybrid electric vehicles', IEEE Trans. Ind. Electron., Vol. 55, No. 6, pp.2237-2245, 2008.

6. M. E. Murphy, 'Neighborhood electric vehicles: the simple things that move you.', Proceedings of NORTHCON'94, 1994.

7. M.E. Murphy, 'A Neighborhood Electric Vehicle (NEV) developed in Oregon for the 21st century.', IEEE Technical Applications Conference. Northcon/96, 1996

8. J. Kim, C. Park, S. Hwang, Y. Hori, and H. Kim, 'Control algorithm for an independent motor-drive vehicle', IEEE Trans. Veh. Technol., Vol. 59, No. 7, pp.3213-3222, 2010.

9. C. H. Lee, K. T. Chau, and L. Cao,'Development of Reliable Gearless Motors for Electric Vehicles', IEEE Trans. Magn., Vol. 5, No. 11, pp.1-8, 2017.

10. Y. E. Zhao, J.W. Zhang, and X. Q. Guan,'Modeling and simulation of electronic differential system for an electric vehicle with two-motorwheel drive', Intell. Veh. Symp., pp.1209-1214, 2009.

11. L. D. Novellis, A. Sorniotti, and P. Gruber,'Design and compar- ison of the handling performance of different electric vehicle lay- outs', Proceedings of the Institution of Mechanical Engineers, Part D: J. of Auto. Engg., Vol. 228, No. 2, pp.218-232, 2014.

12. N. Mutoh, T. Kazama, and K. Takita,'Driving characteristics of an electric vehicle system with independently driven front and rear wheels', IEEE Trans. Ind. Electron., Vol. 53, No. 3, pp.803-813, 2006.

13. Y. Zhao, W. Deng, J. Wu, and R. He,'Torque control allocation based on constrained optimization with regenerative braking for electric vehicles', Int. J. of Automotive Tech., Vol. 18, No. 4, pp.685-698, 2017

14. S. Sharma, R. Pegu, and P. Barman,'Electronic differential for electric vehicle with single wheel reference', Int. conf. Power, Dielectric and Energy Management, pp.1-5, 2015. 
15. J. F. Daya, P. Sanjeevikumar, F. Blaabjerg, P. W. Wheeler, and J. O. Ojo, 'Implementation of wavelet-based robust differential control for electric vehicle application', IEEE Trans. Power Electron., Vol. 30, No. 12, pp.6510-6513, 2015.

16. P. P. J. Francisco, I. Cervantes, and A. Emadi,'Stability of an electric differential for traction applications', IEEE Trans. Veh. Technol., Vol. 58, No. 7, pp.3224-3233, 2009.

17. K. Hartani, Y. Miloud, and A. Miloudi, 'Electric vehicle stability with rear electronic differential traction', Proceed. Int. Symp. on Environment Friendly Energies in Electrical Appl., 2010.

18. S. You, H. Lee, D. Lee, H. Mok, Y. Lee, and S. Han,'Speed ratio control for electronic differentials', IET Electron. Lett., Vol. 47, No. 16, pp.933-934, 2011.

19. N. Mutoh, and T. Takayanagi,'Cornering performance of front and rear wheel independent drive type electric vehicles (FRID EVs) under severe road conditions', IEEE Conf. Intell. Transport. Syst., 2011.

20. R. Krishnan,'Electric motor drives: modeling, analysis, and con- trol', Prentice Hall, 2001.

21. G. Tao, Z. Ma, L. Zhou, and L. Li,'Anovel driving and control system for direct-wheel-driven electric vehicle', IEEE Trans. onMagnet.,Vol. 41,No. 1, pp.497-500, 2004.

22. P. Podder, K. Das, S. Neroula, P. Barman, S. Sharma, 'Development of an automated setup for dynamic evaluation of DC motor characteristics'. In 2017 IEEE Calcutta Conference (CALCON), pp. 190-195, December 2017.

\section{AUTHORS PROFILE}

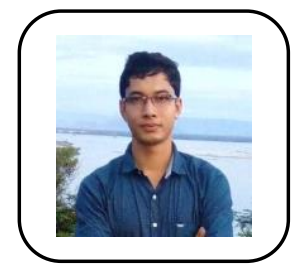

Pranjal Barnman is the research scholar of the Department of Electronics and Communication Engineering. He did his M. Tech in Electronics Design and Technology from Tezpur University, Assam, India. His area of research includes semiconductor devices, power electronics and electric vehicle.

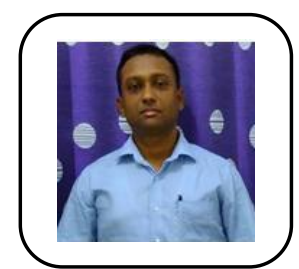

Santanu Sharma received the B.E. degree in electrical engineering from the Jorhat Engineering College, Dibrugarh University, Jorhat, India, in 1998, and the M.Tech. degree in electronics design and technology and the Ph.D. degree in electronics and communication engineering from Tezpur University, Tezpur, India, in 2000 and 2010, respectively. He was a Project Staff Member with the International Centre for Radio Science, Jodhpur, in 2000. He was an Engineering Assistant at Prasar Bharati, Broadcasting Corporation of India, AIR, from 2000 to 2001. From 2001 to 2010, he was an Assistant Professor with the Electronics and Communication Engineering Department, Tezpur University, where he is currently an Associate Professor. His area of research includes semiconductor devices, bioelectronics devices, electric vehicle, and power electronics. 